\title{
Germaine Depierre (2013) Crémation et archéologie. Nouvelles alternatives archéologiques en ostéologie humaine. Éditions universitaires de Dijon, Collection «Art, Archéologie et Patrimoine », Dijon, 654 p
}

\author{
A. Schmitt \\ (C) Société d'Anthropologie de Paris et Lavoisier SAS 2015
}

Suite à l'essor de l'archéologie préventive, les découvertes d'ensembles funéraires livrant des sépultures primaires et/ou secondaires de crémation se multiplient. A contrario, la recherche méthodologique portant sur les restes humains crématisés a connu peu d'évolution depuis l'article de référence de H. Duday, G. Depierre et T. Janin en $2000^{1}$.

La publication d'une partie des travaux réalisés par Germaine Depierre dans le cadre de son doctorat arrive, par conséquent, à point nommé pour dynamiser un pan non négligeable de la recherche archéothanatologique.

La première partie propose de définir un vocabulaire approprié pour ce type de vestiges et fait le point sur les études entreprises dans différentes disciplines afin de répondre à des questions variées (identification, température...) sur les ossements brûlés. De temps à autre, l'auteur développe une discussion sur la nécessité et la difficulté de développer des méthodes appropriées à la restitution des pratiques mortuaires à partir des os brûlés. Elle aborde, par exemple, à propos de l'estimation de l'âge au décès des individus immatures, la relation entre la maturité sociale et la maturité biologique. On est un peu déçu que ce type de

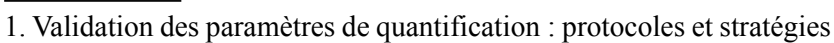
dans l'étude anthropologique des sépultures secondaires à incinération. L'exemple des nécropoles protohistoriques du Midi de la France. In: Archéologie de la mort, archéologie de la tombe au premier âge du fer. Actes du XXI ${ }^{\mathrm{e}}$ Colloque international de l'Association française pour étude de l'âge du fer, Conques-Montrozier, 8-11 mai 1997, thème spécialisé, 2000, pp 7-29.
}

\footnotetext{
A. Schmitt $(\square)$

UMR 7268 - ADES, CNRS, Aix-Marseille Univ., EFS,

faculté de médecine Nord, boulevard Pierre-Dramard,

F-13344 Marseille cedex 15, France

e-mail : aurore.schmitt@univ-amu.fr
}

discussion ne soit pas développé pour les autres paramètres. Je concède, cependant, que l'ouvrage comportant déjà $654 \mathrm{p}$ aurait été tout bonnement intransportable.

La deuxième partie présente des données inédites issues d'observations en crématorium sur le comportement du corps et des ossements pendant la crémation ainsi qu'un nouveau référentiel pondéral de la contribution des différents segments anatomiques au poids moyen du squelette. Jusqu'à présent, ce type de référentiel n'avait jamais été entrepris sur des restes crématisés. Germaine Depierre maîtrise parfaitement les limites inhérentes au matériau de son étude. Les arguments précis et développés qu'elle avance aussi bien que l'efficacité de son protocole, et les résultats obtenus justifient largement ce travail de longue haleine. La communauté scientifique est désormais pourvue de nouvelles données et de nouveaux référentiels qu'elle ne peut ignorer.

La partie suivante contient les résultats de plusieurs recherches qui ne sont pas liées aux données issues du crématorium. Deux volets constituent une avancée non négligeable pour la discipline. D'une part, la création d'un référentiel pondéral à partir de la collection Gemmerich a permis de comparer le poids de sujets traités en crématorium à celui de sujets non brûlés, permettant ainsi de discuter de la pertinence des comparaisons de données pondérales pour l'analyse des restes crématisés en contexte archéologique. D'autre part, l'auteur a cherché dans la littérature et par l'expérimentation sur des ossements issus de séries ostéoarchéologiques des critères pertinents pour distinguer les vestiges osseux issus de la crémation d'un cadavre « frais » d'ossements dits « secs ». Les résultats obtenus sur ce matériel apportent de nouveaux éléments ; ils montrent également que la question est extrêmement complexe et qu'il est avant tout crucial de définir clairement ce qu'est un os sec, 
ce qui constitue un enjeu du futur. Cette partie s'achève sur les critères de détermination des os humains crématisés et fragmentés. Ces données faisaient cruellement défaut aux manuels d'ostéologie et sont par conséquent d'un intérêt majeur. Ceux qui se sont essayés à cet exercice savent que même avec une bonne formation et une longue expérience en identification sur des os non brûlés, les vestiges brûlés nécessitent un apprentissage supplémentaire.

L'intérêt des résultats obtenus et de leur limite par rapport à ce qui existait auparavant constitue la quatrième partie de l'ouvrage qui est clos par une synthèse de ces résultats dans la conclusion.

L'organisation des travaux suit globalement celui du doctorat dont ils sont issus : un état de l'art qui a suscité les nouvelles recherches, une présentation détaillée du matériel, des méthodes et des résultats obtenus, suivie d'une discussion replaçant leur apport et leur limite. Ce choix ne posera pas de problèmes aux spécialistes des crémations qui connaissent déjà les problématiques liées à l'étude des os brûlés. Il est en revanche moins accessible à ceux, notamment aux étudiants, qui débutent leur apprentissage et/ou leur recherche sur ce thème. Il s'agit plus d'un traité comme le souligne H. Duday dans la préface que d'un manuel à proprement parler comme il est qualifié par l'auteur dans l'avertissement.

Quoi qu'il en soit, on ne peut que souligner la rigueur, le perfectionnisme, l'objectivité ainsi que le recul dont l'auteur fait preuve tout au long de l'ouvrage. La démarche, les faiblesses et les biais éventuels sont explicités en détail à chaque étape de la réflexion. Les données sont traitées statistiquement afin de valider leur pertinence, tout en restant tout à fait lucide sur les pièges d'une telle démarche. Les documents proposés dans les annexes permettent d'englober la totalité des données. De nombreux tableaux et graphiques illustrent les démonstrations. Le choix de découper chaque partie en sous-parties rend la lecture très agréable également. Un bémol, cependant : les photographies sont souvent de mauvaise qualité alors qu'elles auraient dû être une valeur ajoutée à l'ouvrage. Les illustrations sur les os brûlés étant plutôt rares dans la littérature scientifique, nous devons saluer l'effort entrepris par l'auteur pour réunir cette documentation et déplorer d'autant son peu de netteté.
Outre un bilan sur les paramètres, les méthodes et les techniques mises à contribution pour l'étude archéothanatologique des os brûlés, l'ouvrage présente de nouvelles données ou précise quelques lieux communs sur le comportement du corps pendant la crémation, apporte de nouveaux référentiels pondéraux, avance des critères permettant de distinguer la crémation d'un cadavre " frais » de la combustion d'«os secs» et propose des méthodes d'identification des ossements brûlés. L'apport de ces travaux est indiscutable et incontournable pour les futures recherches sur les crémations archéologiques. Les biais d'échantillonnage et d'analyse étant communiqués, c'est aux utilisateurs d'exploiter, ou non, selon leurs problématiques, les nouveaux éléments qui sont désormais à leur disposition.

L'ouvrage suscite, par ailleurs, de nombreuses questions. À plusieurs reprises, l'auteure discute de la pertinence de mener certains types d'études en tenant compte de l'équation des paramètres temps-coût-intérêt. Elle insiste sur le fait que les études maximalistes ne sont pas toujours souhaitables, ni même justifiées, et je ne peux qu'abonder en ce sens. Elle aborde également la question de la formation à l'identification des os humains brûlés qui passe par un long apprentissage (« un stage de 15 jours ou de quatre mois ne semble pas suffisant pour travailler en autonomie » p. 517). Que les ossements soient brûlés, ou non, la formation ostéologique doit être rigoureuse puisqu'il s'agit d'une étape cruciale pour toute étude archéothanatologique. Toutefois, la mise en garde énoncée par Germaine Depierre risque d'en décourager beaucoup alors que les demandes d'études d'os brûlés ne cessent de se multiplier. Le contexte actuel de la recherche et de l'archéologie préventive ne se prête guère à ce type de formations au long cours. Si les organismes de formation peuvent susciter des vocations, ils ne peuvent assumer l'intégralité d'un tel apprentissage. Il est donc temps de s'interroger sur cette spécialité et de trouver des solutions appropriées. Sans cela, la recherche sur les os crématisés permettant d'en tirer le meilleur parti scientifique risque de se scléroser alors que, comme le conclut Germaine Depierre, ce champ d'investigation est non seulement vaste mais les progrès méthodologiques tout à fait réalisables, comme elle a su le démontrer tout au long de son ouvrage. 